\title{
Surgical Treatment in Patients with Cervical Osteomyelitis: Single Institute's Experiences
}

\author{
Bang Sang Hahn, Kyung-Hyun Kim, Sung-Uk Kuh, Jung Yoon Park, \\ Dong-Kyu Chin, Keun-Su Kim, Yong-Eun Cho \\ Department of Neurosurgery, The Spine and Spinal Cord Institute, Gangnam Severance Spine Hospital, \\ Yonsei University College of Medicine, Seoul, Korea
}

\begin{abstract}
Objective: To study practical guidelines and strategies in the treatment of cervical osteomyelitis. Methods: We retrospectively reviewed 14 patients who underwent surgical treatment for cervical osteomyelitis from May 2000 to July 2008 . We investigated their clinical course, antibiotic regimen, surgical methods, and laboratory and radiologic findings including X-ray, CT and MRI.

Results: 5 patients had primary spondylodiscitis, 5 patients had post operative spondylodiscitis and 4 patients had tuberculosis in cervical spine. The causative microorganisms were MRSA(5), P. aeruginosa (1), Methicillin resistant coagulase negative streptococcus (1), P. aeruginosa changed to MRSA(1), and 2 patients showed no growth on culture studies. Patients were treated 13.8 weeks (range, 5.4-25.8) with IV antibiotics and then treated for 58.2 days (range, 13-106) with oral antibiotics. Antituberculotic medications were used for a mean of 383.8 days. Patients were treated with anterior debridement and fusion (5), irrigation and debridement (5), simultaneous cervical anterior interbody and transthoracic thoracic interbody fusion (1). 3 patients underwent the planned 2-staged operation, which included an anterior debridement with or without fusion for the $1^{\text {st }}$ operation and posterior instrumentation for $2^{\text {nd }}$ operation. 10 patients $(71.4 \%)$ had neurologic deficits at the time of diagnosis and 7 patients (70\%) among them improved post-operatively.

Conclusion: Anterior cervical spine surgery is the preferable treatment option in patients with neurological deterioration, extensive bony destruction with expected kyphotic deformity, and uncontrolled infection being managed only with antibiotics. Antibiotics are also important for thorough treatment.
\end{abstract}

Key Words: Cervical spine $\cdot$ Spinal infection $\cdot$ Osteomyelitis $\cdot$ Spondylodiscitis

\section{INTRODUCTION}

Osteomyelitis of the spine is a rare disease accounting for approximately 1 to $7 \%$ of all bone infections, and only 3 to $6 \%$ of spinal osteomyelitis is confined to the cervical spine ${ }^{19)}$. Because of the large diameter of the cervical cord in relative to the spinal canal and the significant range of motion, even a small epidural abscess can cause devastating results ${ }^{1}$.

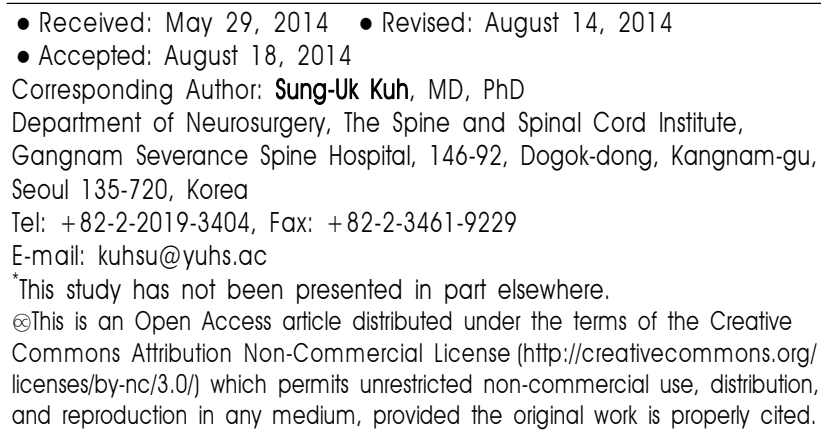

Bryan Barnes et al. published a thorough Meta-Analysis of cervical osteomyelitis cases, conducting a most comprehensive literature-based review of the etiology, diagnosis, and treatment of cervical vertebral osteomyelitis $(\mathrm{CVO})^{2}$. The authors listed alcoholism, dental work, liver disease, diabetes, previous history of surgery, drug abuse, and tuberculosis as a medical comorbidities or serious medical illness predisposing CVO and reported that number of patients requiring surgical intervention ranged from 18 to $43 \%$ (without distinction of cervical or non-cervical), and among surgically treated patients, most preferred approach was direct anterior debridement. After debridement, no further treatment or anterior fusion, anterior and posterior fusion were considered as a further treatment options. However, although standard management for cervical vertebral osteomyelitis is medical treatment using proper antibiotics, this article did not proposed practical guidelines depend on each patients with cervical osteomyelitis which should be required surgical treatment, in that there are various surgical strategy and options to choose especially in cervical osteomyelitis because of several factors such as degree of the 
infectious progress, site of spinal cord compression, and expected kyphotic deformity due to bony destruction or disc space involvement.

Thus we analyzed clinical cases of cervical osteomyelitis treated in the single institute, which were caused by various causative factors, and studied the strategic factors that should be considered. Also, we studied treatment results of antibiotics after operation for cervical osteomyelitis.

\section{MATERIALS AND METHODS}

We retrospectively reviewed 14 patients who had been treated surgically for cervical osteomyelitis from May 2000 to July 2008 (Table 1). We investigated their clinical course, comorbid medical diseases, antibiotic regimen, surgical methods, and their laboratory and radiologic findings including plain X-ray, computed tomography (CT), magnetic resonance imaging (MRI) and clinical outcomes (Preoperative and post-operative Visual Analogue Scale (VAS) score, American Spinal Injury Association (ASIA) impairment scale).

In most cases, the diagnosis of cervical osteomyelitis can be accomplished by using plain X-ray films and computerized tomography scans. Nevertheless, preferential use of T1 gadolinium enhanced magnetic resonance imaging is helpful in identifying epidural compressive processes in patients suffered from neurological deficit. In our study, all patients were evaluated with MRI to confirm diagnosis, additionally using CT images for estimating bony destruction and instability. Also, laboratory results such as WBC with differential counting, ESR (erythrocyte sedimentation rate), CRP (C-reactive protein) was performed for confirmation of diagnosis, severity of disease, clinical response to antibiotics, and used as guideline for decision to discontinue antibiotics ${ }^{11)}$.

Indications for surgical treatment included neurological deficit due to spinal cord compression, progressive deformity and instability of the cervical spine, intractable pain despite of conservative treatment, poorly controlled infectious condition with antibiotics administration.

\section{RESULTS}

\section{The Preoperative Conditions}

This study included ten men and four women, whose mean

Table 1. Preoperative demographics of the cervical osteomyelitis patients

\begin{tabular}{|c|c|c|c|c|c|c|c|c|}
\hline Case & $\begin{array}{c}\text { Age (yrs)/ } \\
\text { Sex }\end{array}$ & Etiology & $\begin{array}{c}\text { Micro } \\
\text { organism }\end{array}$ & Medication & Presenting symptom & Fever & $\begin{array}{l}\text { Swallowing } \\
\text { difficulty }\end{array}$ & $\begin{array}{l}\text { Risk } \\
\text { factor }\end{array}$ \\
\hline 1 & $52 / \mathrm{M}$ & Spontaneous & MRSA & VM & Quadriplegia & N & N & $\mathrm{DM}$ \\
\hline 2 & $72 / \mathrm{M}$ & Spontaneous & P. aeruginosa & $3^{\text {rd }}$ Cefa + AMG & PNP and radiculopathy & N & N & \\
\hline 3 & $50 / M$ & Spontaneous & MRSA & $3^{\text {rd }}$ Cefa+VM & PNP and radiculopathy & Y & N & \\
\hline 4 & $56 / F$ & Spontaneous & No growth & $3^{\text {rd }}$ Cefa + AMG & PNP and radiculopathy & N & $\mathrm{N}$ & \\
\hline 5 & $56 / M$ & Spontaneous & MRSA & $\mathrm{VM}+\mathrm{AMG}$ & PNP and radiculopathy & Y & $\mathrm{N}$ & \\
\hline 6 & $21 / M$ & $\mathrm{Tbc}$ & $\begin{array}{l}\text { Mycobacterium } \\
\text { tuberculosis }\end{array}$ & HERZ & PNP and radiculopathy & Y & Y & \\
\hline 7 & $69 / \mathrm{M}$ & Tbc & $\begin{array}{l}\text { Mycobacterium } \\
\text { tuberculosis }\end{array}$ & HERZ & Paraparesis & N & N & \\
\hline 8 & $26 / F$ & Tbc & $\begin{array}{l}\text { Mycobacterium } \\
\text { tuberculosis }\end{array}$ & HERZ & PNP & Y & N & \\
\hline 9 & $56 / F$ & Tbc & $\begin{array}{l}\text { Mycobacterium } \\
\text { tuberculosis }\end{array}$ & HERZ & PNP and radiculopathy & N & N & \\
\hline 10 & $43 / M$ & Postop & MRSA & $3^{\text {rd }} \mathrm{Cefa}+\mathrm{VM}+\mathrm{AMG}+\mathrm{CM}$ & Pus discharge & Y & Y & \\
\hline 11 & $30 / M$ & Postop & $\begin{array}{l}\text { P. aeruginosa } \\
\rightarrow \text { MRSA }\end{array}$ & $3^{\text {rd }}$ Cefa $+\mathrm{AMG} \rightarrow \mathrm{VM}$ & Pus discharge & Y & Y & \\
\hline 12 & $59 / \mathrm{M}$ & Postop & MRSA & VM & Wound swelling & Y & N & $\mathrm{DM}$ \\
\hline 13 & $44 / F$ & Postop & MRCNS & $\mathrm{VM}+\mathrm{AMG}$ & Neck swelling & N & N & \\
\hline 14 & $49 / \mathrm{M}$ & Postop & No growth & VM & PNP & N & Y & DM \\
\hline
\end{tabular}


age was 48.8 (range, 21-69) years old. 5 patients had primary spontaneous spondylodiscitis, 5 patients had post operative spondylodiscitis and 4 patients had tuberculosis in cervical spine.

The average symptom durations were 9 days for primary pyogenic spondylodiscitis and 21.5 days for tuberculosis in the cervical spine. In post operative spondylodiscitis, the revision surgery was done in 14.8 days after 1st operation. The average hospital stay was 62 (range, 17-349) days. The causative microorganisms in pyogenic infection were Methicillin resistant S. aureus (5), P. aeruginosa (1), Methicillin resistant coagulase negative streptococcus (1), P. aeruginosa changed to MRSA (1) and 2 patients showed no growth on culture studies. Mycobacterium tuberculosis was confirmed in all tuberculosis patients.

The patients enrolled in this study had no risk factors such as immunocompromised status, intravenous drug user, or have undergone an oropharyngeal procedure except for 3 patients with diabetes mellitus and 2 patients who suffered esophageal injury during cervical anterior interbody fusion surgery.

7 patients experienced fever and 4 patients had swallowing difficulty. $10(71.4 \%)$ patients had preoperative neurological deficit. $7(70 \%)$ patients among them improved post-operatively (Table 1).

\section{Medical Treatment}

The antibiotics were administered a mean of 38.6 (range, 23-75) days with culture sensitive intravenous (IV) antibiotics and then were treated a mean of 58.2 (range, 13-106) days with oral antibiotics for primary pyogenic spondylodiscitis. However, patients with spondylodiscitis were treated longer, as follows: mean 21 (6.6-48.6) weeks with culture sensitive IV antibiotics and then 43.3 (9-70) days with oral antibiotics. Generally, IV antibiotics was replaced with oral antibiotics, which was mostly $2^{\text {nd }}$ generation cephalosporin, if serum CRP levels were maintained below $5 \mathrm{mg} / \mathrm{L}$ for 4 weeks and the ESR and white blood cell (WBC) count were within normal limits. IV antibiotics covering anaerobes were also administered to post-operative infected patients, especially in case of esophageal perforation.

In the 4 cases of post-tuberculosis infections, we used an antituberculotic regimen (isoniazid; INH $(\mathrm{H})$, ethambutol; EMB (E), rifampicin; RMP (R), pyrazinamide; PZA (Z), HERZ) for a mean 383.8 days.

\section{Surgical Treatments}

We classified spinal lesion types as follows: cord compression without deformity (2), kyphotic deformity without cord compression (3), both spinal cord compression and kyphotic deformity (6), and neither cord compression or kyphotic deformity (3). Eleven patients underwent single stage surgical treatment including cervical anterior interbody fusion with AIBG (5), infection site debridement with or without irrigation catheter insertion (5), and simultaneous performed cervical anterior interbody fusion at the C5-6-7-T1 levels and transthoracic thoracic interbody fusion at the T5-6-7 levels with

Table 2. Surgical results of the cervical osteomyelitis patients

\begin{tabular}{|c|c|c|c|c|c|c|c|}
\hline Case & $\begin{array}{c}\mathrm{F} / \mathrm{U} \\
\text { (months) }\end{array}$ & $\begin{array}{l}\text { Type of } \\
\text { Lesion }\end{array}$ & $\begin{array}{c}\text { Involved } \\
\text { level }\end{array}$ & Operation method & $\begin{array}{c}\text { ASIA } \\
\text { (Preoperative/Final) }\end{array}$ & $\begin{array}{c}\text { VAS } \\
\text { (Preop/Postop) }\end{array}$ & Complications \\
\hline 1 & 9.6 & $\mathrm{C}(+), \mathrm{K}(+)$ & $\mathrm{C} 6 / 7$ & 2 stages operation (CAIF, PI) & $\mathrm{B} / \mathrm{D}$ & $9 / 2$ & \\
\hline 2 & 13.8 & $C(-), K(+)$ & $\mathrm{C} 3 / 4$ & CAIF & $E / E$ & $7 / 1$ & \\
\hline 3 & 10.6 & $\mathrm{C}(+), \mathrm{K}(+)$ & $\mathrm{C} 5 / 6$ & CAIF & $C / D$ & $9 / 3$ & \\
\hline 4 & 2.1 & $C(-), K(-)$ & $\mathrm{C} 5 / 6$ & CAIF & $E / E$ & $8 / 2$ & \\
\hline 5 & 19.6 & $C(+), K(+)$ & $\mathrm{C} 5-\mathrm{Tl}$ & CAIF & $E / E$ & $7 / 1$ & \\
\hline 6 & 7.1 & $\mathrm{C}(-), \mathrm{K}(+)$ & $\mathrm{Cl}-2$ & 2 stages operation $(\mathrm{I} \& \mathrm{D}, \mathrm{PI})$ & $C / D$ & $8 / 2$ & \\
\hline 7 & 10.3 & $\mathrm{C}(+), \mathrm{K}(+)$ & C5-6 & 2 stages operation (CAIF, PI) & $D / E$ & $7 / 1$ & \\
\hline 8 & 13 & $\mathrm{C}(+), \mathrm{K}(+)$ & $\mathrm{C} 7, \mathrm{~T} 7$ & CAIF \& TAIF & $C / D$ & $7 / 1$ & \\
\hline 9 & 10.2 & $\mathrm{C}(+), \mathrm{K}(-)$ & $\mathrm{C} 3 / 4 / 5$ & CAIF & $E / E$ & $8 / 2$ & \\
\hline 10 & 34.5 & $C(+), K(+)$ & $C 5 / 6 / 7$ & $1 \& D$ & $\mathrm{D} / \mathrm{D}$ & $9 / 7$ & Hoarseness/Kyphosis \\
\hline 11 & 4.7 & $C(+), K(-)$ & $\mathrm{C} 4 / 5 / 6 / 7$ & $1 \& D$ & A/A & $9 / 6$ & Hardware Failure \\
\hline 12 & 37.9 & $C(-), K(-)$ & $\mathrm{C} 4 / 5$ & $1 \& D$ & $\mathrm{~A} / \mathrm{A}$ & $9 / 4$ & \\
\hline 13 & 25.5 & $C(-), K(-)$ & $\mathrm{C} 4-\mathrm{Tl}$ & $1 \& D$ & $C / D$ & $8 / 3$ & \\
\hline 14 & 4.2 & $\mathrm{C}(-), \mathrm{K}(+)$ & $C 4 / 5 / 6$ & $1 \& D$ & $C / D$ & $9 / 2$ & \\
\hline
\end{tabular}

F/U, Period of follow-up; C, Cord compression; K, Kyphotic deformity; CAIF, Cervical anterior interbody fusion; PI, Posterior Instrumentation; I\&D, Incision and Debridement. 
AIBG and plate reinforcement for cervicothoracic tuberculosis (1). The 2 stage operation included 1 case of anterior debridement and 2 cases of anterior debridement with AIF at the time of first operation and posterior fixation with instruments was performed during the second operation (Table 2).

In one patient with post-operative infection after cervical AIF C4-5-6-7 due to esophageal rupture (case 11), follow up $\mathrm{x}$-ray showed fracture of the previously inserted anterior cervical plate after anterior debridement for infection control. Therefore, we removed the fractured anterior cervical plate because solid fusion was done already. In the other patient (case 10) who had an intraoperative esophageal rupture and extensive mediastinitis, we could not perform the $2^{\text {nd }}$ operation for stabilization due to continuous pus discharge and uncontrollable infection after $1^{\text {st }}$ operation for esophageal repair and debridement. Thus, kyphotic deformity with cord compression developed 5 months after the 1st operation, but the infection was finally cured with antibiotics.

$10(71.4 \%)$ patients had neurologic deficits at the time of diagnosis and 7 patients (70\%) among them improved postoperatively.

\section{DISCUSSION}

Cervical spine spondylodiscitis is a rare, but serious manifestation of spinal infection ${ }^{9}$. In cervical vertebral osteomyelitis and abscess, it can be more rapid deteriorating processes causing neurologic deficit ${ }^{14,21)}$. Thus, the surgical strategy should focus on spinal biomechanical stabilization, neural decompression, and infectious tissue removal ${ }^{14,19)}$. There are various predisposing risk factors for spondylodiscitis which include diabetes mellitus, rheumatic diseases, immunosuppressive diseases, previous invasive treatment modalities (pharyngeal surgery, tonsillectomy etc), septic condition (pneumonia, urinary tract infection, etc), and intravenous drug abuse $e^{3,4,5,6,15,20,22)}$. In our series, there were 3 patients with diabetes mellitus and 2 patients with esophageal injuries during cervical anterior surgery.

\section{Medical Treatment}

There are no solid guidelines for antibiotics for cervical osteomyelitis, but generally a minimum of 6 to 8 weeks is sufficient for pyogenic infections ${ }^{17)}$. The average duration of IV antibiotic treatment for post-operative infections was 21 (range, 6.6-48.6) weeks. It was definitely a longer duration than previous reports because of the small sample size, which included some chronic uncontrolled infections. A standard antibiotic treatment regimen cannot be derived from published results and the choice of regimen usually depends on clinician's preferences or policy ${ }^{13,16}$. Since we inference that a patient had spondylitis through his or her clinical symptoms, imaging studies, and laboratory results, we usually began broad spectrum IV antibiotics such as a combination of third generation cephalosporin, penicillin with $\beta$-lactamase inhibitor, and aminoglycoside before the culture sensitivity was reported. We switched from IV antibiotics to oral antibiotics if the serum CRP level was maintained below $5 \mathrm{mg} / \mathrm{L}$ for 4 weeks. And we used an oral antibiotic for 1 to 2 months for complete infection control.

\section{Preoperative Surgical Planning and Strategy}

\section{1) Preoperative Clinical General Condition}

In cases of elderly patients with poor general conditions or rapid neurological deterioration or a chronic uncontrolled infectious process, we preferred to plan a staged operation to reduce perioperative morbidities. First an operation was performed for neural decompression or early identification of the causative organism and infection control. Then, a second operation for stabilization or preventing an expected kyphotic deformity was performed as soon as possible.

In elderly patients, reduced operative times and intraoperative blood loss during each operation can diminish the perioperative complication ${ }^{10)}$.

\section{2) Exact Extent of Infection Involvement in Spondylitis for Surgical Planning}

Because unnecessary excessive corpectomies could cause iatrogenic instability and result in unneeded secondary operations for stabilization or higher complication rate in multi-segmental fusions ${ }^{8,18)}$, meticulous preoperative interpretation of both T1 gadolinium enhanced MRI and sagittal reconstruction CT scans should be necessary to determine the exact extent of infection involvement. It is important to distinguish infection involvement from simple bone swelling in order to decide the corpectomy level.

In case 1 (Fig. 1), T1 gadolinium enhanced MR images revealed whole vertebral enhancement but sagittal reconstruction CT scan showed that bony destruction was limited to the lower part of the C6 vertebrae and the upper part of the C7 vertebrae. Therefore, we decided to perform segmental cervical anterior debridement and fusion with an autologous bone graft at the C6/7 level. In contrast, both T1 gadolinium MRI and sagittal reconstruction CT scan exhibited extensive bony destruction, especially middle column of the C5 and $\mathrm{C} 6$ in case 7 (Fig. 2). Therefore, we should perform corpectomies both at the C5 and C6 vertebrae. 


\section{3) Esophageal Injury Especially in Post-operative Spondylitis}

In post-operative infection cases, it is important to check the esophageal injury. It can be detected by esophagography and neck or chest CT with contrast media ${ }^{23)}$. If there is an esophageal injury, we chose primary esophageal repair, irrigation, debridement, and silastic or catheter drainage for $1^{\text {st }}$ operation and used broad spectrum antibiotics with the addition

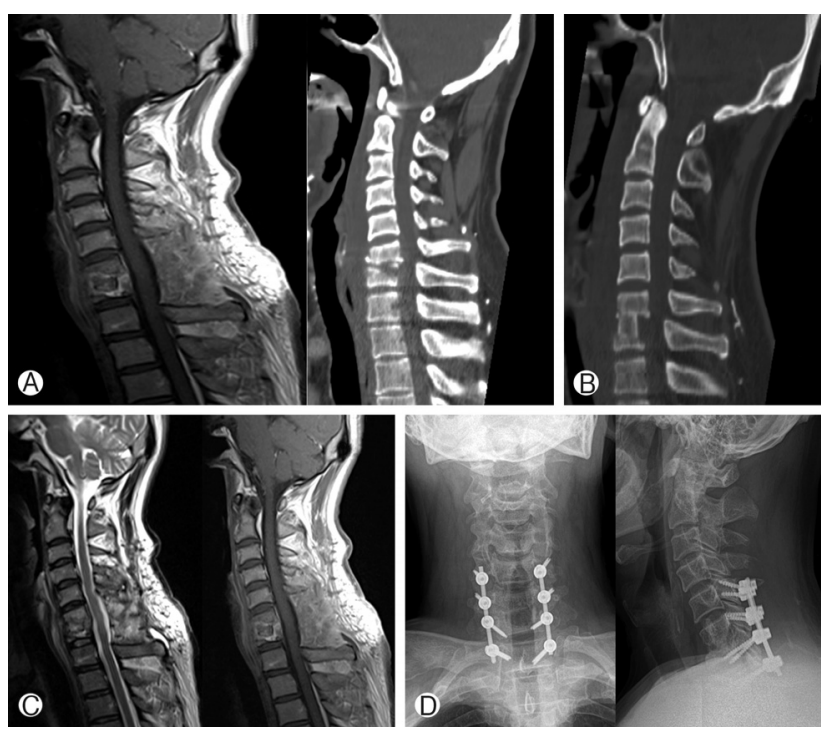

Fig. 1. 52 year-old male (case 1) who underwent debridement, discectomy and fusion with iliac bone at the C6-7 segment first, then, posterior lateral mass screw fixation for the second operation. MRI and CT before the first operation showed disc space disruption and relatively sparing of vertebral body, mostly inflammatory swelling (A). CT scan after the $1^{\text {st }}$ operation (B), MRI after the $2^{\text {nd }}$ operation $(C)$, plain $X$-ray after $2^{\text {nd }}$ operation $(D)$. of anaerobes-covering antibiotics such as clindamycin or metronidazole simultaneously. The esophagography should be followed-up regularly until it is confirmed that there is no leakage from the repair site. Then, a secondary operation for stabilization should be planned as soon as possible because an extensive infectious process can cause devastating results such as progressive kyphotic deformity with cord compression (case 10, Fig. 3).

\section{4) Preexisting Instability or Expected Kyphotic Deformity}

Anterior debridement and fusion has advantages for relieving neurological deterioration and ceasing the infectious process in the early stage. In patients with epidural or paravertebral abscesses compressing the cord but no preexisting instability, we prefer to choose the anterior approach without posterior stabilization for debridement and fusion if indicated. However, if there is extensive bony destruction and disc space invasion causing instability, we believe that three column stabilization should be performed by combined anterior fusion and posterior fixation ${ }^{7}$.

The combined approach also minimizes pseudoarthrosis, graft dislodgement, deformity progression, and anterior plate failure which contributes to worse prognosis in complicated pathological disorders like multicolumn involvement or extensive multilevel invasion with infection ${ }^{12)}$.

\section{5) Limitation}

First of all, the number of patient who was enrolled in this study was too small and heterogeneously mixed with pathogen to draw conclusion. Surgical discipline for tuberculosis and bacterial disease should be differentiated because bony
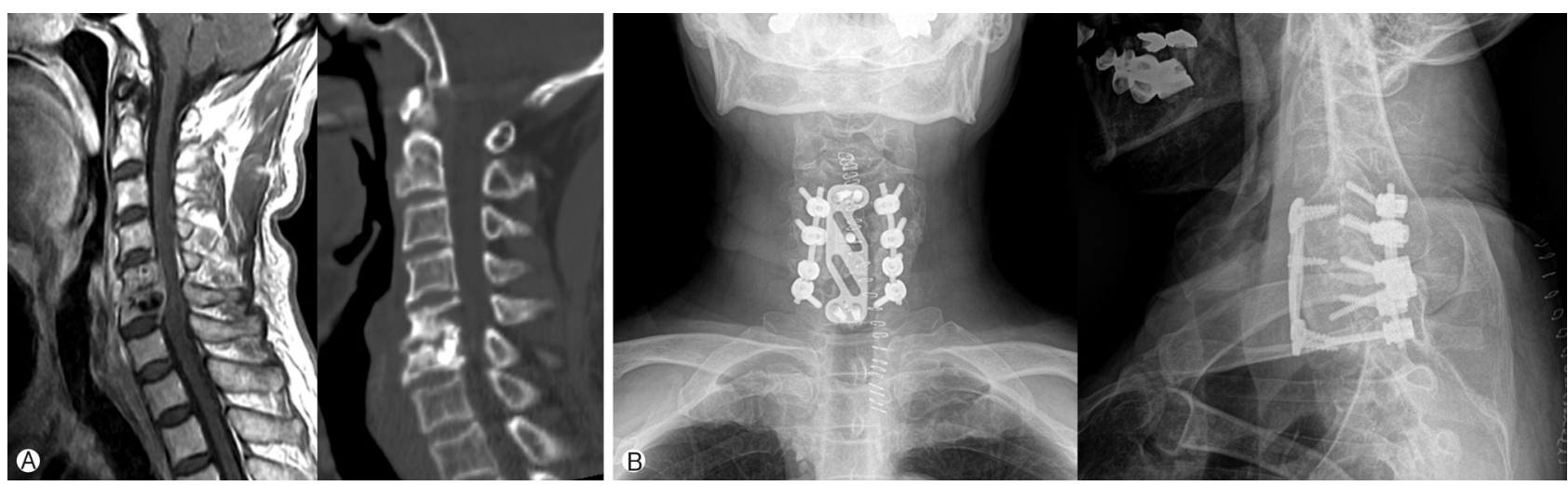

Fig. 2. 69 year-old male (case 7) who initially underwent corpectomy of C5 and C6 and fusion of C4-7 segments with iliac bone and plate. Then, posterior lateral mass screw fixation at the C4-5-6-7 segments for the second operation. Preoperative $\mathrm{T} 1$ enhanced MRI and CT revealed extensive bony destruction, especially at the middle column (A). 14 days after the $1^{\text {st }}$ operation (B). 

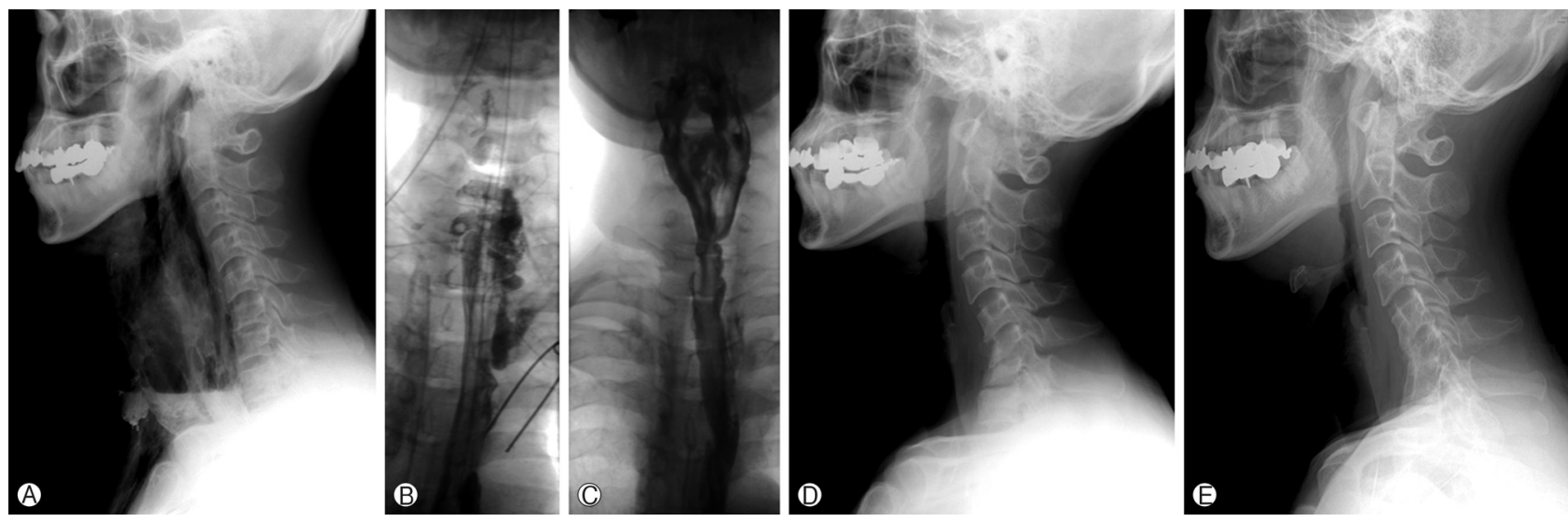

Fig. 3. 43 year-old male (case 10) presented greenish discharge 2 days after ACDF C6/7. Initial C-spine X-ray (A) and esophagography (B) showed barium and contrast media leakage into the mediastinum. Follow-up esophagography (C) 1 month after primary repair revealed no leakage from repair site. Kyphotic deformity progressed on post-operative 5 months X-ray (D). X-ray at post operative 34.5 months showed bony fusion at the C5-6-7 segments with severe kyphotic angulation (E)

destruction process by tuberculosis could be different from bacterial origin. However as mentioned earlier in this literature, cervical osteomyelitis is a rare disease and also the portion of patients demanding surgical treatment is small. Further study including larger group of patients would be necessary for more reliable conclusion.

This study consisted of primary and secondary (postoperative) infections, also bacterial and non-bacterial (tuberculosis) infections, lacking consistency. On the other hand, heterogeneity of subjects made in possible to analysis variable aspect of cervical osteomyelitis and their surgical management to each situation. We, in this article, want to discuss or investigate the detailed surgical techniques including approach, level of corpectomy, and special consideration in overall. For example, distinct characteristics of tuberculous osteomyelitis are that more preserved bony quality, relative disc preservation, and more prominent extra-vertebral abscess, which needs distinguished strategies in surgical decision making.

\section{CONCLUSION}

Anterior cervical spine surgery is the preferable treatment option in patients with neurological deterioration, extensive bony destruction with expected kyphotic deformity, and uncontrolled infection being managed only with antibiotics. The anterior-posterior combined approach, which combines the anterior approach for rapid neural decompression and identification of causative microorganism and the posterior approach for stabilization of the preexisting instability or expected kyphotic deformity, could be a preferable choice for multilevel diseases. Also, sufficient and proper uses of antibiotics with follow-up of laboratory results are important for thorough treatment of cervical osteomyelitis

\section{REFERENCES}

1. Acosta FL, Jr., Chin CT, Quinones-Hinojosa A, Ames CP, Weinstein PR, Chou D: Diagnosis and management of adult pyogenic osteomyelitis of the cervical spine. Neurosurg Focus 17:E2, 2004

2. Barnes B, Alexander JT, Branch CL Jr.: Cervical osteomyelitis: a brief review. Neurosurg Focus 17(6):E11, 2004

3. Boutoille D, Talarmin JP, Prendki V, Raffi F: Hematogenous anaerobic vertebral osteomyelitis due to Bacteroides fragilis in a diabetic patient. Eur J Intern Med 14:63-64, 2003

4. Brown DL, Musher DM, Taffet GE: Hematogenously acquired Aspergillus vertebral osteomyelitis in seemingly immunocompetent drug addicts. West J Med 147(1):84-85, 1987

5. Calvo JM, Ramos JL, Garcia F, Bureo JC, Bureo P, Perez M: Pyogenic and non-pyogenic vertebral osteomyelitis: descriptive and comparative study of a series of 40 cases. Enferm Infecc Microbiol Clin 18:452-456, 2004

6. Endress C, Guyot DR, Fata J, Salciccioli G: Cervical osteomyelitis due to i.v. heroin use: radiologic findings in 14 patients. AJR Am J Roentgenol 155(2):333-335, 1990

7. Gok B, Sciubba DM, McLoughlin GS, McGirt M, Ayhan S, Wolinsky JP, et al: Surgical treatment of cervical spondylotic myelopathy with anterior compression: a review of 67 cases. J Neurosurg Spine 9(2):152-157, 2008

8. Gore DR: The arthrodesis rate in multilevel anterior cervical fusions using autogenous fibula. Spine 26(11):1259-1263, 2001

9. Heyde CE, Boehm H, El Saghir H, Tschoke SK, Kayser R: Surgical treatment of spondylodiscitis in the cervical spine: a minimum 2-year follow-up. Eur Spine J 15:1380-1387, 2006

10. Kawaguchi Y, Kanamori M, Ishihara H, Ohmori K, Abe Y, Kimura T: Pathomechanism of myelopathy and surgical results of laminoplasty in elderly patients with cervical spondylosis. Spine 28:2209-2214, 2003 
11. Ki-Hyun Jeon, Jin-Young Youm, Seung-Won Choi, Hyun-Song Koh, Shi-Hun Song, Youn Kim: Significance of Changes in Erythrocyte Sedimentation Rate and C-Reactive Protein Counts in Pyogenic Spondylitis Treatment. Korean J Spine 11(1):507512, 2004

12. Kim PK, Alexander JT: Indications for circumferential surgery for cervical Spondylotic myelopathy. Spine J 6:299S-307S, 2006

13. Leys D, Lesoin F, Viaud C, Pasquier F, Rousseaux M, Jomin M: Decreased morbidity from acute bacterial spinal epidural abscesses using computed tomography and nonsurgical treatment in selected patients. Ann Neurol 17:350-355, 1985

14. Nakase H, Matsuda R, Tamaki R, Tei R, Park YS, Sakaki T: Two-stage management for vertebral osteomyelitis and epidural abscess: technical note. Neurosurgery 58:E1219; discussion E 1219, 2006

15. Patel AA, Madigan L, Poelstra KA, Whang PG, Vaccaro AR, Harrop JS: Acute cervical osteomyelitis and prevertebral abscess after routine tonsillectomy. Spine J 8:827-830, 2008

16. Reihsaus E, Waldbaur H, Seeling W: Spinal epidural abscess: a meta-analysis of 915 patients. Neurosurg Rev 23(4):175-204, 2000
17. Rezai AR, Woo HH, Errico TJ, Cooper PR: Contemporary management of spinal osteomyelitis. Neurosurgery 44:10181025; discussion 1025-1026, 1999

18. Saunders RL, Pikus HJ, Ball P: Four-level cervical corpectomy. Spine 23:2455-2461, 1998

19. Schimmer RC, Jeanneret C, Nunley PD, Jeanneret B: Osteomyelitis of the cervical spine: a potentially dramatic disease. J Spinal Disord Tech 15:110-117, 2002

20. Silvani V, Brambilla G, Rainoldi F, Gaetani P, Denaro V: Vertebral osteomyelitis with chronic cervical extradural abscess in a heroin addict. Neurochirurgia (Stuttg) 30:91-94, 1987

21. Spuck S, Arnold H, Kranz R, Solbach W, Kaemmerer R: Rapid manifestation of cervical vertebral osteomyelitis. Acta Neurochir (Wien) 147:671-673; discussion 673, 2005

22. Walters HL, Measley R: Two cases of Pseudomonas aeruginosa epidural abscesses and cervical osteomyelitis after dental extractions. Spine 33:E293-296, 2008

23. Witwer BP, Resnick DK: Delayed esophageal injury without instrumentation failure: complication of anterior cervical instrumentation. J Spinal Disord Tech 16:519-523, 2003 